\title{
EFEITOS DO TEMPO DE FREQÜÊNCIA DO PRÉ-ESCOLAR AO PROGRAMA CENTRO DE EDUCAÇÃO E ALIMENTAÇÃO DO PRE-ESCOLAR SOBRE O DESEMPENHO AVALIADO PELO “INSTRUMENTO II", ADICIONAL *
}

\begin{abstract}
FERNANDES, J. \& GANDRA, Y. R. Efentos do tempo de frequêencia do pré-escolar do Programa Centro de Educacão e Alimentasão do Pré-Escolar sobre o desen. penho avaliado pelo "Instrumento II', adicional Rer. Saúde públ., S. Paulo, 15 (supl.) :105-15, 1981 .
\end{abstract}

RESUMO: Estudada a influência do tempo de freqüência dos pré-escolares ao programa CEAPE sobre o desempenho de algumas tarefas cognitivas, perceptivas e de linguagem quando analisadas pelo "Instrumento Adicional de Avaliação do Desenvolvimento de Pré-Escolares" (IADPE-II). Os pré-escolares de 5 a 6 anos de idade, recém ingressados (" $R$ ") e com mais de seis meses de frequiência ("A") foram testados e retestados após um intervalo de 6 meses letivos. Os resultados obtidos com os testes iniciais discriminaram significantemente estes dois grupos de pré-escolares. O estudo dos incrementos obtidos quando avaliados pelo "Reteste" indicou que todos os grupos de estudo apresentaram incrementos significantes, embora os grupos recém ingressados " $R$ " tenderam a obter maiores progressos que os respectivos grupos iniciais antigos " $\mathrm{A}$ ". Os incrementos nos resultados obtidos pelo pré-escolar são maiores nos 6-7 meses letivos iniciais, o que poderá sugerir a necessidade da reestruturação das atividades de Recreação orientada em termos de ordenação crescente de aquisição mais complexa.

UNITERMOS: Pré-escolares, avaliação. Criança, desenvolvimento. Programa CEAPE.

\section{INTRODUÇÃO}

A idade pré-escolar constitui um periodo de grande elaboração, a nível físico e psicológico ${ }^{6}$. Neste período, acentua-se ainda mais a importância de dois aspectos básicos e relacionados: alimentação adequada e estimulação ambiental a determinados processos psicológicos (cognitivos, perceptivos e outros).
O desenvolvimento físico e psicológico ocorrido no período pré-escolar fornecerá os subsídios para outros importantes eventos futuros, tal como a escolarização. Entretanto, nem sempre a família do pré-escolar - especialmente as de baixo nível sócio-econômico - tem possibilidade de satisfazer plenamente estes aspectos levantados.

* Convènio $10 / 77$ - INAN/DN/FSP/USP.

* Do Programa CEAPE - Departamento de Nutrição da Faculdade de Saúde Pública da USP - Av. Dr. Arnaldo, 715 - 01255 - São Fa'lo, SP - Brasil.

*** Do Departamento de Nutrição da Faculdade de Saúde Pública da USP - Av. Dr. Arnaldo, 715 - 01255 - São Paulo, SP - Brasil. 
FE:RNANDES, J. \& GANDRA, Y.R. Efeitos do tempo de frequiencia do pré-escolar do Programa Centro de Educação e Alimentação do Pré-Escolar sobre o desempenho avaliado pelo "Instrumento II", adicional. Rev. Saủảe Públ., S Paulo, 15(supl.): 105-15, 1981.

Uma das linhas de atuação, nesse sentido, é a implantação de programas que ofereçam ao pré-escolar suplementação alimentar e a estimulação de certos processos psicológicos tal como ocorre com o programa CEAPE - Centros de Educação e Alimentação do Pré-Escolar (Gandra, 1973) ${ }^{4.5}$.

O objetivo da recreação orientada desse programa é a estimulação das áreas cognitivas, perceptivas, motora, de expressão e comunicação social. A flexibilidade do programa e a utilização de recursos comunitários ociosos possibilitaram a expansão do CEAPE a diversas comunidades do interior do Estado de São Paulo e a implantação de programas semelhantes, em outros estados 10.

O processo de avaliação psico-pedagógica do programa CEAPE foi iniciado através do "Instrumento de Avaliação do Desenvolvimento do Pré-Escolar - IADPE”2, que é um procedimento simples, de avaliação breve de aspectos tidos como importantes na análise do desenvolvimento da criança. $O$ instrumento revelou diferenças altamente significativas entre crianças "Ceapenses" e "Não-Ceapenses", de mesma idade e da mesma comunidade. Os resultados obtidos também mostraram que este instrumento foi nos dois grupos altamente sensivel aos efeitos da idade; embora para as crianças "Ceapenses", a partir de 5 anos, o teste tendesse, como era de se esperar, a diminuir o seu grau de discriminação.

Em vista deste último resultado, foi elaborado o "Instrumento II", adicional ", para complementar o "IADPE", na avaliação de crianças de 5 e 6 anos. Este instrumento segue as mesmas características gerais do "IADPE" por ser também de testagem breve, pouco sofisticada. As análises iniciais deste instrumento revelaram que ele discriminou significativamente entre crianças "Ceapenses" de 5 e de 6 anos de idade.

Com base nestes resultados, o objetivo do presente trabalho é efetuar uma estimativa inicial da influência do tempo de fre- qüència ao programa CEAPE sobre o desempenho em algumas tarefas cognitivas, perceptivas e de linguagem, conforme avaliadas pelo "Instrumento II", adicional.

\section{MATERIAL E MÉTODOS \\ ist....}

Os trabalhos de avaliação psicopedagógica foram centralizados na cidade de Leme, que contava, no inicio desta pesquisa, com cerca de 12 CEAPEs em funcionamento, sob orientação educacional comum, fornecida diretamente pela Prefeitura local. Em Leme, todos os CEAPEs funcionam em locais fechados E cobertos, durante cerca de 4 horas diárias. A admissão ao programa é aberta a pré-escolares de qualquer nível sócio-econômico, embora a população assistida seja a de baixo nivel sócio-econômico.

Cada centro atende de 30 a 40 crianças. Todas as crianças recebem suplementação alimentar (merenda) e atividades psicopedagógicas, na forma de Recreação Orientada (jogos, dramatizações, pintura, colagem, recorte, entre outras). O objetivo da Recreação Orientada é a estimulação rotativa das áreas cognitiva, perceptiva, motora, de expressão e comunicação social e de conhecimento sobre o meio ${ }^{1}$.

$\mathrm{O}$ atendimento às crianças é realizado por professoras primárias, não necessariamente especializadas em pré-escolares. As mães dos pré-escolares também participam do programa em um esquema de rodízio, desenvolvendo atividades relacionadas com a recreação e/ou merenda, sob a orientação da responsável. O "Instrumento II" oferece uma avaliação breve, de aplicação individual e suas caracteristicas foram discutidas em trabalho anterior ${ }^{3}$.

$O$ presente trabalho pretende verificar 0 efeito do tempo de frequiência dos pré-escolares ao CEAPE, sobre o desempenho frente ao "Instrumento II". Todas as crianças foram avaliadas por uma equipe constituida por elementos previamente treinados e padronizados. Outros detalhes do procedimento são discutidos em trabalho anterior ${ }^{3}$. 
F'ERNANDES, J. \& GANDRA, Y.R. Efeitos do tempo de frequiencia do pré-escolar do Programa Centro de Educação e Alimentação do Pré-Escolar sobre 0 desempenho avaliado pelo "Instrumento II", adicional. Rev. Saude Públ,, S. Paulo, 15(supl.): 105-15, 1981.

Os testes foram executados em duas ocasiões diferentes (teste inicial em abril e reteste em outubro do mesmo ano). O reteste foi realizado com algumas das crianças que passaram pelo teste inicial. O intervalo teste inicial-reteste corresponde a cerca de 5 meses letivos.

No teste "inicial" 3 foram formados 4 grupos, constituidos por crianças "Ceapenses" de 5 ou 6 anos. Para cada idade, foram formados 2 grupos: um de crianças recém-ingressas ao programa CEAPE (freqüência menor ou igual a 2 meses) e outro, por crianças com, no mínimo, 6 meses consecutivos de frequiência, com uma percentagem de comparecimento de, pelo menos, $75 \%$. Os grupos de crianças recém-ingressas ao programa serão designados por $5 \mathrm{R}$ e $6 \mathrm{R}$ (para 5 e 6 anos, respectivamente) e os grupos de crianças "antigas" ao programa, por $5 \mathrm{~A}$ e $6 \mathrm{~A}$.
A distribuição do número de crianças em cada grupo, a freqüência média em meses letivos de cada grupo ao programa, assim como as médias, desvios-padrões amostrais e as medianas das idades, em meses, para cada grupo, encontra-se em trabalho anterior ${ }^{3}$. As diferenças de idade, entre " $5 R$ " e " $5 \mathrm{~A}$ "; " $6 \mathrm{R}$ " e " $6 \mathrm{~A}$ " não são significativas ao nivel de $5 \%$, bicaudal.

As Tabelas 1 a 9 referem-se a dados coletados em entrevista, com a mãe ou responsável pela criança. Os dados de uma mesma família foram computados apenas uma vez. Assim, o número de familias é menor que o número de crianças testadas, dada a existência de irmãos entre elas.

Pode-se notar pela Tabela 1 que, de um modo geral, o nível de escolaridade dos país e das mães situou-se entre o curso "primário incompleto" e o "primário completo".

T A B E L A 1

Nível de escolaridade das mãe e dos pais dos pré-escolares testados do Programa CEAPE - Municipio de Leme, SP*.

\begin{tabular}{|c|c|c|c|c|}
\hline \multirow{2}{*}{ Nivel de escolaridade } & \multicolumn{2}{|c|}{ Mães } & \multicolumn{2}{|c|}{ Pais } \\
\hline & $N^{9}$ & $\%$ & No & $\%$ \\
\hline Sem instruçãa & 18 & 7,41 & 15 & 6,30 \\
\hline $\begin{array}{c}1-3 \\
\text { (primário incompleto) }\end{array}$ & 96 & 39,51 & 94 & 40,34 \\
\hline $\begin{array}{c}4-5 \\
\text { (primário completo) }\end{array}$ & 113 & 46,50 & 102 & 44,54 \\
\hline $\begin{array}{c}6-7 \\
\text { (ginasial incompleto) }\end{array}$ & 8 & 3,29 & 10 & 4,20 \\
\hline $\begin{array}{c}8-9 \\
\text { (ginasial completo) }\end{array}$ & 5 & 2,06 & 10 & 4,20 \\
\hline $\begin{array}{c}10 \text { ou }+ \\
\text { (colegial ou }+ \text { ) }\end{array}$ & 3 & 1,23 & 7 & 2,94 \\
\hline Total & 243 & - & 238 & - \\
\hline Sem informação & 28 & - & 33 & - \\
\hline
\end{tabular}

* Adotou-se a nomenclatura vigente na ocasião em que as pessoas entrevistadas estudaram. 
FERNANDES, J. \& GANDRA, Y.R. Efeitos do tempo de freqüência do pré-escolar do Programa Centro de Educação e Alimentação do Pré-Escolar sobre o desempenho avaliado pelo

"Instrumento II", adicional. Rev. Saúde Públ., S. Paulo, 15(supl.) : 105-15, 1981.

As famílias de crianças "Ceapenses", no geral, podem ser classificadas como sendo de baixa renda (cerca de $63 \%$ das familias foram classificadas na faixa de 2 a 5 salários mínimos de renda mensal bruta) (Tabela 2).

T A B E L A 2

Renda familiar mensal bruta, declarada em entrevista, em termos de salários mínimos, para todos os grupos de pré-escolares testados, do Programa CEAPE - Município de Leme, SP.

\begin{tabular}{lrr}
\hline \multicolumn{1}{c}{ Renda } & No & $\%$ \\
\hline $0\lceil-1$ sal. mínimo & 5 & 2,07 \\
$1-2$ sal. mínimos & 40 & 16,60 \\
$2\lceil-3$ sal. mínimos & 56 & 23,24 \\
$3\lceil-5$ sal. mínimos & 95 & 39,42 \\
$5\lceil-7$ sal. mínimos & 33 & 13,69 \\
7 ou + sal. mínimos & 12 & 4,98 \\
\hline Total & 241 & - \\
\hline Sem Informação & 30 & - \\
\hline
\end{tabular}

Conforme mostra a Tabela 3, o tamanho da familia nuclear das crianças "Ceapenses" variou em $\mathbf{7 2 \%}$ dos casos, entre 3 e 6 pessoas.

T A B E L A 3

Tamanho da família nuclear, para todos os grupos de pré-escolares testados, do Programa CEAPE - Município de Leme, SP.

\begin{tabular}{|c|c|c|}
\hline $\begin{array}{l}\text { Tamanho } \\
\text { da familia }\end{array}$ & No da família & $\%$ \\
\hline $3|-| \quad 4$ & 102 & 37,64 \\
\hline $5|-| 6$ & 92 & 33,95 \\
\hline $7|-| \quad 8$ & 34 & 12,55 \\
\hline $9 \mid-10$ & 14 & 5,17 \\
\hline 11 ou + & 1 & 0,37 \\
\hline Total & 243 & - \\
\hline Sem Informação & 28 & - \\
\hline
\end{tabular}

A Tabela 4 apresenta a renda famíliar mensal bruta per capita, em termos de salários mínimos. Pode-se norta que em cerca de $80 \%$ das familias, a renda mensal per capita variou entre zero e um salário mínimo, havendo uma maior concentração de casos na faixa entre meio e un salário minimo de renda mensal per capita.

\section{T A B E L A 4}

Renda familiar mensal bruta per capita, em termos de salários mínimos, para todos os grupos de pré-escolares testados, do Programa CEAPE - Município de Leme, SP.

\begin{tabular}{lrc}
\hline \multicolumn{1}{c}{$\begin{array}{c}\text { Renda } \\
\text { familiar mensal per capita }\end{array}$} & $\mathrm{N}^{\circ}$ & $\%$ \\
\hline $0 \quad$ - 0,5 sal. mínimo & 66 & 27,85 \\
$0,5\lceil$ 1,0 sal. mínimo & 127 & 53,59 \\
$1.0\lceil-1,5$ sal. mínimos & 37 & 15,65 \\
$1,5\lceil-2,0$ sal. mínimos & 6 & 2,53 \\
$2,0 \mid-2,5$ sal. mínimos & 0 & - \\
2,5 ou + sal. mínimos & 1 & 0,42 \\
\hline Total & 237 & - \\
\hline Sem Informação & 34 & - \\
\hline
\end{tabular}

A Tabela 5 mostra o nível de Prestigio ocupacional do chefe da família, segundo a Escala de Hutchinson modificada por Gouveia (1970) ${ }^{\tau}$. As classificações foram feitas com base nos critérios de Macedo $(1973)^{9}$. A grande maioria das profissões relatadas pode ser classificada nos níveis mais baixos da escala, com predominânciá de ocupações manuais especializadas e semelhantes (mecânicos, torneiros, eletricistas, entre outras). Resumindo pode-se dizer que as crianças "Ceapenses" testadas sãn provenientes, de um modo geral, de famílias de baixo nível sócio-econômico em termos de renda, escolaridade dos pais e nível de prestígio ocupacional do chefe da família. 
FERNANDES, J. \& GANDRA, Y.R. Efeitos do tempo de frequência do pré-escolar do Programa Centro de Educação e Alimentação do Pré-Escolar sobre o desempenho avaliado pelo "Instrumento II", adicional. Rev. Saúde Públ., S Paulo, 15(supl.): 105-15, 1981.

\section{T A B E L A 5}

Nivel de prestígio ocupacional do chefe de família, com base na escala de Hutchinson, modificada por Gouveia*, para as famílias dos pré-escolares testados, do Programa CEAPE - Mrnicípio de Leme, SP.

\begin{tabular}{lcc}
\hline \multicolumn{1}{c}{ Níveis da escala ** } & Ne & $\% 6,86$ \\
\hline $\begin{array}{l}\text { 1. Ocupações manuais não especializadas } \\
\text { 2. Ocupações manuais especializadas e } \\
\text { semelhantes }\end{array}$ & 87 & 51,27 \\
$\begin{array}{l}\text { 3. Supervisão de trabalho manual e } \\
\text { semelhantes }\end{array}$ & 121 & $\mathbf{1 , 2 7}$ \\
$\begin{array}{l}\text { 4. Ocupaçóes não manuais de rotina e } \\
\text { semelhantes }\end{array}$ & 3 & 4,66 \\
$\begin{array}{l}\text { 5. Proprietários de pequenas empresas } \\
\text { comerciais e semelhantes } \\
\text { Aposentados }\end{array}$ & 6 & 2,54 \\
\hline $\begin{array}{l}\text { Total } \\
\text { Sem Informação }\end{array}$ & 836 & 3,39 \\
\hline Gouveia (1970) & 35 & - \\
As profissóes foram classificadas com base & nos critérios de Macedo (1973)9,
\end{tabular}

Para se proceder ao "Reteste", algumas crianças de cada um dos quatro grupos citados acima $(5 \mathrm{R}, 5 \mathrm{~A}, 6 \mathrm{R}$ e $6 \mathrm{~A})$ foram retestadas 6 meses após o teste "Inicial". Este intervalo de tempo correspondeu, na realidade, a cerca de 5 meses de atividades efetivas. Os pré-escolares de 5 anos de idade que passaram pelo "Reteste" ("grupos de seguimento") serão denominados S5R e S5A (respectivamente "recém-ingressas" e "antigas" ao programa, na ocasião do teste "Inicial") e, da mesma forma, S6R e S6A, para crianças de 6 anos (Tabela 6).

A Tabela 7 apresenta as médias, desvios-padrões amostrais e as medianas das idades, em meses, para cada grupo de seguimento, na ocasião do teste "Inicial". As diferenças de idade entre "S5R e S5A"; "S6R e S6A" não são significativas, a um nivel de $5 \%$, bicaudal.

T A B E L A 6

Número e freqüência média de pré-escolares ao Programa CEAPE (em meses letivos), em abril e outubro do mesmo ano, para cada grupo de seguimento - Município de Leme, SP.

\begin{tabular}{lcccc}
\hline \multicolumn{1}{c}{ Gripo } & S5R & S5A & S6R & S6A \\
\hline No & 44 & 13 & 31 & 19 \\
Freq. - abril & 2 & 11 & 2 & 13 \\
Freq. - out. & 7 & 16 & 7 & 18 \\
\hline
\end{tabular}


FERNANDES, J. \& GANDRA, Y.R. Efeitos do tempo de frequência do pré-escolar do Programa Centro de Educação e Alimentação do Pré-Escolar sobre o desempenho avaliado pelo

"Instrumento II", adicional. Rev. Saúde Públ., S. Paulo, 15(supl.): 105-15, 1981.

\section{TA B E L A 7}

Médias, desvios-padrões amostrais e medianas das idades, em meses, no início do estudo (abril), para cada grupo de pré-escolares do Programa CEAPE - Municipio de Leme, SP*.

\begin{tabular}{lc|cccc}
\hline $\begin{array}{l}\text { Idade } \\
\text { (abril) }\end{array}$ & Gripo & S5R & S5A & S6R & S6A \\
\hline Média & 66 & 65 & 75 & 75 \\
Desvio-padrão & 3,29 & 3,61 & 2,15 & 2,14 \\
Mediana & 67 & 65 & 75 & 74 \\
\hline
\end{tabular}

* As diferenças entre os grupos de mesma idade não são significativas, ao nivel de $\mathbf{5} \%$, bicaudal.

\section{RESULTADOS}

Os resultados obtidos no total do "Instrumento II" e no total das suas diferentes áreas serão analisados separadamente.

Os resultados do teste "Inicial" que serviu de base ao seguimento foram apresentados em trabalho anterior ${ }^{3}$ quando se comparou "Ceapenses" recém ingressados no programa, " $R$ ", com os que já o freqüentaram há mais de 6 meses, " $A$ ".

A Figura 1, apresenta os totais médios de cada área, para cada grupo, expressos em percentagens do total máximo de pontos da área, assim como os valores de $Z$ para as comparações " $R$ " $X$ " $A$ ", entre grupos de mesma idade, com as suas respectivas significâncias. Como vimos, os grupos "A" obtiveram resultados sistematicamente mais elevados que os respectivos grupos " $R$ ", de mesma idade, em todas as áreas. Isto revela que as diferenças " $R$ " $X$ " $A$ ", no total do "Instrumento II", refletiram diferenças de desempenho ao longo de todo o teste.

Foram encontradas diferenças significativas entre os grupos "R" - "A", aos 5 e 6 anos, nas áreas 1 - DISCR e III COR e, aos 5 anos, para as áreas IV FUNC e V - VERB. As diferenças "R" -. "A" da área II - PERC não foram significativas aos 5 ou 6 anos, o que sugere que o programa CEAPE (Leme) aparentemente não conseguiu escimular suficientemente esta área.
Pode-se notar que as diversas áreas do Instrumento detectaram mais diferenças significativas "R" e "A" aos 5 do que aos 6 anos. A falta de significância das diferenças "R" - "A", aos 6 anos, em 3 das 5 áreas do "Instrumento II", sugere hipoteses diferentes e não mutuamente exclusivas:

a) os efeitos do programa CEAPE tendem a ser maiores aos 5 do que aos 6 anos em algumas das áreas avaliadas pelo teste (especialmente as IV - FUNC. e V - VERB.; já que II - PERC. não apresentou diferenças significativas aos 5 ou 6 anos);

b) a falta de significância pode ser atribuída a características intrínsecas do teste. Por exemplo, IV - FUNC. e $\mathrm{V}$ - VERB., conforme foi levantado, não discriminaram efetivamente entre os grupos 5A e $6 \mathrm{~A}$ (ou seja, estas áreas deixam de ser sensiveis a efeitos de idade, após um certo tempo de frequiência do programa). Isto parece indicar que estas áreas apresentam limitações para a avaliação de grupos "A", o que contribuiria para os resultados obtidos.

Dessa forma, a questão de qual a faixa etária mais influenciada pelo ingresso ao programa ( 5 ou 6 anos) permanece aberta, merecendo novos estudos, não se podendo ainda fazer afirmações conclusivas a respeito. 
FERNANDES, J. \& GANDRA, Y.R. Efeitos do tempo de freqüencia do pré-escolar do Programa Centro de Educação e Alimentação do Pré-Escolar sobre o desempenho avaliado pelo "Instrumento II", adicional. Rev. Saúde Públ., S. Paulo, 15(supl.): 105-15, 1981.

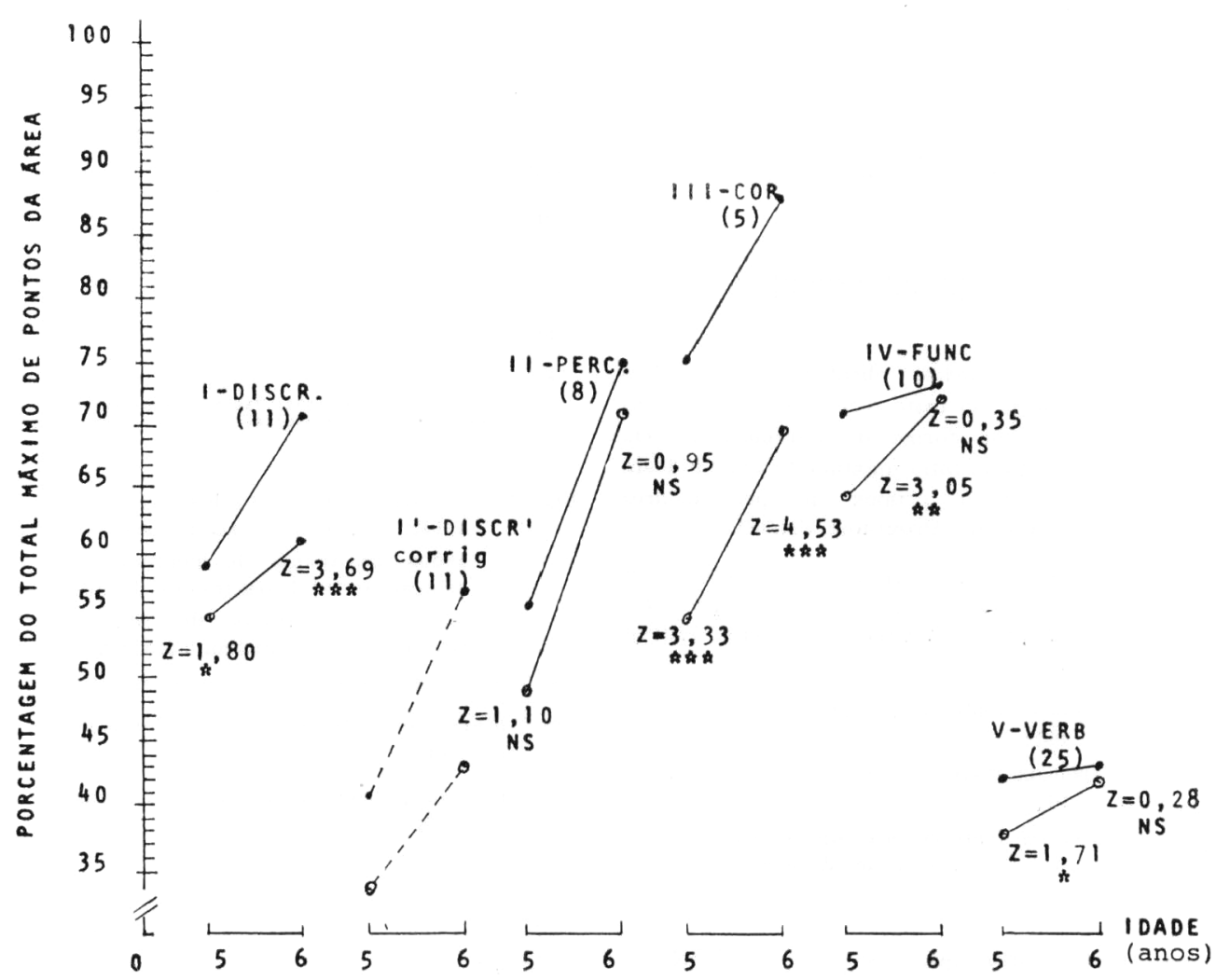

Fig. 1 - Médias de pontos obtidos em cada uma das áreas do Instrumento II adicional, expressas em percentagem do total máximo de pontos da área para os grupos " $R$ " e "A" do Programa CEAPE - Área 1 (corrigida) - DISCR., segundo Guilford 8.

Obs.: Os valores de $Z$ ("R" $x$ "A") foram obtidos através da prova de Mann-Whitney.

$$
\begin{array}{rl}
* \cdot & \mathrm{p}<.05 \\
* * & \mathrm{p}<.01 \\
* * * & \mathrm{p}<.001
\end{array}
$$

NS: diferenças não significativas.

O Reteste objetivou analisar os incrementos obtidos pelos pré-escolares antigos, "A" ou recém ingressados, 6 meses após o teste "Inicial", quando algumas crianças de cada um dos 4 grupos originais foram retestados. Este intervalo de tempo corresponde a cerca de 5 meses letivos.

a) Resultados nas Areas do "Instrumento II" - As Figuras 2 a 6 apresentam os totais médios obtidos em cada área, por grupo de seguimento, na ocasião do teste
"Inicial" (abril) e no "Reteste" (outubro), na forma de percentagens do total máximo de pontos desta área. Os resultados de cada grupo no teste "Inicial" e no "Reteste" foram comparados através da prova não-paramétrica de Wilcoxon (Siegel, 1975)11.

A Figura 2 apresenta os resultados obtidos pelos grupos na área I - DISCR. (sem a introdução da correção de acertos por acaso). 
FERNANDES, J. \& GANDRA, Y.R. Efeitos do tempo de frequêencia do pré-escolar do Programa Centro de Educação e Alimentação do Prê-Escolar sobre o desempenho avaliado pelo "Instrumento II", adicional. Rev. Saúde Públ., S. Paulo, 15(supl.): 105-15, 1981.

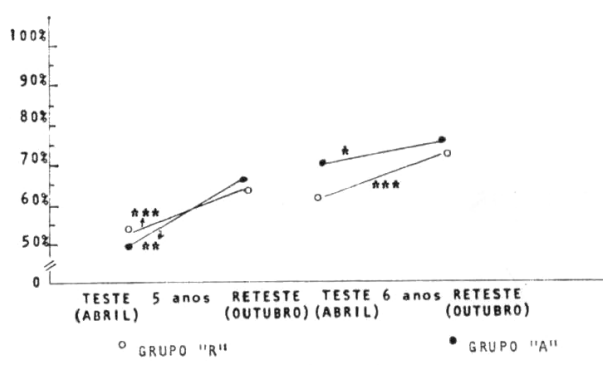

Fig. 2 - Totais médios obtidos na área I DISCR. no teste e no reteste, apresentados na forma de percentagens em relação ao total máximo de pontos desta área para grupos de pré-escolares testados do Programa CEAPE.

Obs.: Grupos " $R$ ", com frequiència média $\leqslant 2$ meses letivos no teste inicial; grupos "A" com frequiencia média $\geqslant 6$ meses letivos no teste inicial;

*i*: diferenças teste-reteste significativas a $\mathrm{p}<.001$;

**; diferenças teste-reteste significativas a $p<.01$;

*: diferenças teste-reteste significativas a $p<.05$, pela prova de Wilcoxon.

Pode-se notar que os grupos "S5R" e "S6R" obtiveram incrementos mais significativos que os respectivos grupos "A", entre o teste "Inicial" e o "Reteste". Além disso, as diferenças existentes entre os grupos S6R e S6A praticamente desapareceram no "Reteste", embora, nesta ocasião, - grupo S6A tenha mais de que o dobro da frequêencia em meses letivos ao programa, que o grupo S6R.

As mesmas tendèncias foram verificadas nas áreas II - PERC. e III - COR, conforme mostram as Figuras 3 e 4.

Novamente, os grupos S5R e S6R obtiveram incrementos significativos entre o teste e o reteste, o mesmo não acontecendo com os respectivos grupos "A". Após um intervalo de 5 meses letivos, as diferenças entre os grupos " $R$ " e "A" diminuiram sensivelmente.

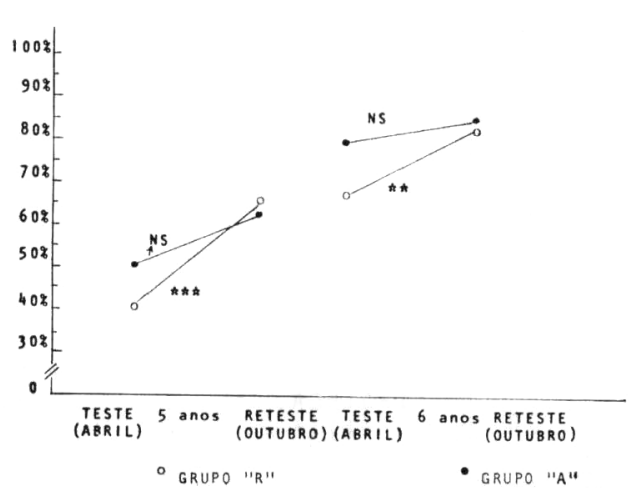

Eig. 3 - Totais médios obtidos na área II PERC. no teste e no reteste, apresentados na forma de percentagens em relação ao total máximo cle pontos desta área para grupos de pré-escolares testados do Programa CEAPE.

NS: diferenças teste-reteste não significativas

**: diferenças teste-reteste significativas a $\mathrm{p}<.01$ :

***: diferenças teste-reteste significativas a p <.001;

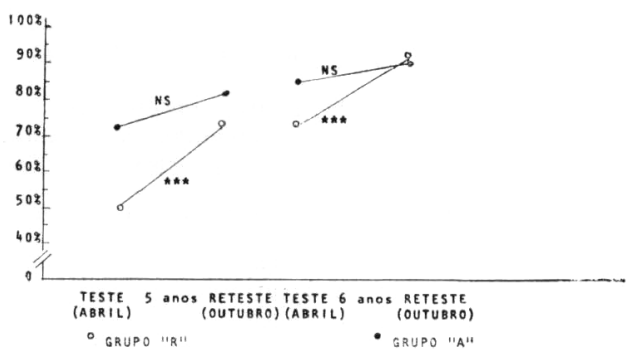

Fig. 4 - Totais médios obtidos na área III COR, no teste e no reteste, na forma de percentagens em relação ao total máximo de pontos desta área para os grupos de pré-escolares testados do Programa CEAPE.

NS: diferenças teste-reteste não significativas ***: diferenças teste-reteste significativas a $p<.001$

Os resultados nas áreas IV - FUNC. e $\mathrm{V}$ - VERB., estão representädos nas Figuras 5 e 6. 
FERNANDES, J. \& GANDRA. Y.R. Efeitos do tempo de frequència do pré-escolar do Programa Centro de Educação e Alimentação do Pré-Escolar sobre o desempenho araliato pelo "Instrumento II", adicional, Rev. Saúde Públ., S Paulo, 15(supl.): 105-15, 1981.

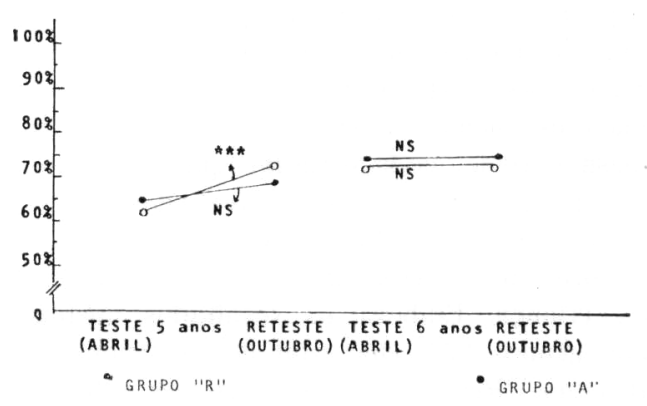

Fig. 5 - Totais médios obtidos na área TV . FUNC. no teste e no reteste, na forma de percentagens em relação ao total máximo de pontos desta área para os grupos de pré-escolares testados do Programa CEAPE.

NS: diferenças teste-reteste não significativas

$\because$ diferencas teste-reteste significativas a $p<.001$;

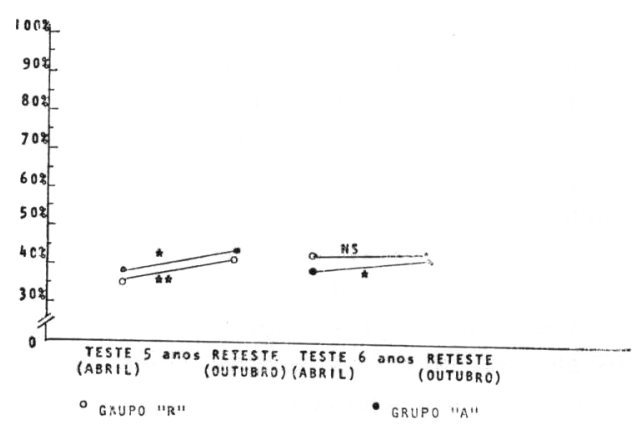

Fig. 6 - Totais médios obtidos na área $\mathrm{V}$. VERB. no teste e no reteste. na forma de percentagens em relação ao totai máximo de pontos desta área para os grupos de pré-escolares testados do Programa CEAPE.

NS: diferenças teste-reteste não significativas diferencas teste-reteste significativas a $p<.01$;

Pode-se notar que as diferenças entre idades e entre grupos " $\mathrm{R}$ " — "A" já estão bastante pequenas, mesmo no teste inicial, o que novamente sugere o pequeno grau de discriminação destas áreas. Estas diferenças praticamente desapareceram na ocasião do reteste. Mesmo assim, o grupo S5R apresentou incrementos significativos nestas duas áreas, enquanto que os grupos S5A e S6A apresentaram incrementos significativos apenas na área $\mathrm{V}$ - VERB.

b) Total do "Instrumento II" - A Figura 7 apresenta os totais médios do "Instrumento II", para os quatro grupos originais do "Teste Inicial" (5R, 5A, 6R, 6A) e para os grupos de seguimento nas duas ocasiões de avaliação. Os totais médios são apresentados na forma de percentagens do total máximo de pontos do teste (59 pontos)

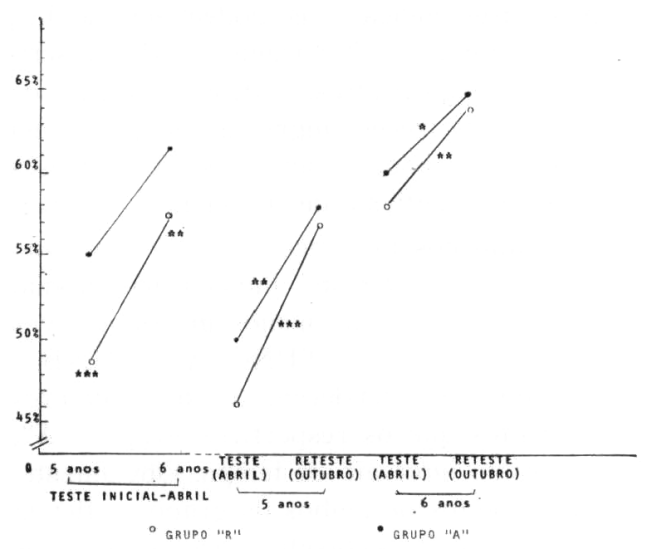

Fig. 7 - Resultados médios obtidos no total do "Instrumento II". na forma de percentagem do total máximo de pontos do teste para os grupos de pré-escolares testados do Programa CEAPE.

Obs.: As comparaçóes " $\mathrm{R}$ " $\mathrm{x}$ "A", nos grupos do teste inicial - abril foram efetuadas através da prova de Mann-Whitney; as comparaçōes entre teste-reteste, em cada grupo, foram realizadas através da prova de Wilcoxon.

*- diferenças teste-reteste significativas a $\mathrm{p}<.001$

**: diferenças teste-reteste significativas a $p<.01$;

*: diferenças teste-reteste significativas a $\mathrm{p}<.05$, pela prova de Wilcoxon 
IERNANDES, J. \& GANDRA, Y.R. Efeitos do tempo de frequiencia do pré-escolar do Programa Centro de Educação e Alimentação do Prí-Escolar sobre o desempenho avaliado pelo "Instrumento II", adicional. Rev. Saúde Públ., S. Paulo, 15(supl.): 105-15, 1981.

Enıbora os grupos de seguimento fossem derivados dos grupos do teste "Inicial", os seus resultados médios (na primeira avaliação) foram, no geral, inferiores aos dos respectivos grupos de origem. Todos os grupos de seguimento apresentaram algum grau de incremento significativo entre as duas ocasiões de avaliação. Entretanto, os ganhos médios foram maiores para os grupos " $R$ ", dentro de uma mesma idade. Novamente nota-se que com relação ao "Total", na ccasião de reteste, foram bastante pequenas as diferenças "R" — "A", dentro de grupos de mesma idade.

\section{DISCUSSÃO E CONCLUSÕES}

Os resultados obtidos até agora, embora preliminares, indicam que podem ser obtidas, como o total do "Instrumento II", discriminações significativas entre crianças de mesma idade, recém ingressas ao programa CEAPE e crianças com mais de 6 meses letivos consecutivos de frequiencia a ele ${ }^{3}$.

$\mathrm{O}$ estudo dos incrementos obtidos quando analisado pelo "Reteste" indicou que, mesmo excluindo-se as áreas menos discriminativas do instrumento (IV - FUNC.; V - VERB.). os grupos "R" tenderam a obter maiores incrementos que os respectivos grupos "A", num mesmo intervalo de tempo. Obviamente, os incrementos de ambos os grupos refletem tanto os efeitos psicopedagógicos específicos do programa CEAPE como também outros aspectos mais gerais de desenvolvimento que ocorreram no intervalo teste-reteste. Supondo-se esta última classe de fatores mais ou menos constante, para grupos de mesma idade, pode-se notar que os efeitos do programa, conforme avaliados pelos incrementos no "Instrumento II", foram maiores nos meses iniciais de freqüencia da criança. Embora os resultados médios dos grupos "A" (no total do "Instrumento II") no "Reteste" fossem maiores que os dos respectivos grupos " $\mathrm{R}$ ", as diferenças entre eles foram muito pequenas (cerca de meio ponto) e muito provavelmente tem pouco ou nenhum significado prático. Isto sugeria que a "Recreação Orientada", aparentemente após cerca de 7 meses letivos, tenderia a atingir um limite de eficácia psicopedagógica, quando avaliados pelo "Instrumento II". Como conseqüência, os incrementos nos resultados do "Instrumento II" obtidos pela criança são maiores nos $6-7$ meses letivos iniciais.

Os atuais resultados sugerem a necessidade de reestruturação das atividades de Recreação Orientada (em termos de delimitação de objetivos operacionalizáveis; ordenaçāo das atividades em função dos objetivos propostos; eventual divisão por faixa etária, entre outras) de tal forma que as habilidades apresentadas pela criança possam ser utilizadas sempre como ponto de partida para aquisições mais complexas.

FERNANDES, J. \& GANDRA, Y. R. [The effects of the length of attendance of the preschool child at the SEAPE Program on performance as evaluated by the additional method of assessment ("Instrument II")], Rev. Satide públ., S. Paulo, 15 (suppl.):105-15, 1981.

ABSTRAC $\Gamma$ : The influence of the length of attendance at the CEAPE program is studied on the cognitive, perceptive and linguistic abilities of the preschool child when these abilities were analysed by "Instrument II". Preschool children from 5 to 6 years old recently admitted (" $R$ ") to the program and those with more than 6 months of attendance ("A"), were tested and retested after 6 months. The first tests differentiated significantly between the two groups of children "A" $x$ "R". The "re-test" after 6 months, shows that all the groups had progressed significantly but the groups of preschool children recently admitted (" $R$ ") to the program demonstrated greater progress than the group "A". The progress achieved was greater within the first six-month period than in the subsequent one. This perhaps suggests, that the activities of the Guided Recreation plan should be restructured in order to achieve more complex acquisition of abilities.

UNITERMS: Preschool, child, evaluation. Child development. CEAPE Program. 
FFRNANDES, J. \& GANDRA, Y.R. Efeitos do tempo de frequiencia do pré-escolar do Programa Centro de Educação e Alimentação do Pré-Escolar sobre o desempenho avaliado pelo "Instrumento II", adicional. Rev. Saúde Públ., S. Paulo, 15(supl.): 105-15, 1981.

\section{REFERENCIAS FIBLIOGRAFICAS}

1. CAVALCANTI, M.L.F. et al. Plano rotativo de recreação orientada. Bol. Ofic. $O M E P$, Rio de Janeiro, (3):5.10, maio/ /jłn. 1976.

2. FERNANDES, J. \& GANDRA, Y.R. Instrumento de avaliação do desenvolvimento de pré-escolares dos Centros de Educação e Alimentação do Pré-Escolar. Rev. Saúde públ., S. Paulo, 15(sıpl.): 79-90, 1981.

3. FERNANDES, J. \& GANDRA, Y.R. "Instrumento II" adicional de avaliação do desenvolvimento de pré-escolares - seu valor na discriminação de grupos etários. Rev. Saúde públ., S. Paulo, 15(supl.): 91-104, 1981.

4 GANDRA, Y.R. Asistencia alimentaria por medio de centros de educación y alimentación del pre-escolar. Bol. Ofic. sanit. panamer., 74:302-14, 1973.

5. GANDRA, Y.R. Nutrition and dietetic eclucation program for preschool children at primary schools in Brazil. In: Anderson, M.A. \& Grewal, T., ed. Nutrition planning in developing world: proceedings of Regional Workshop held by $C A R E$ in India, Kenya and Colombia, 1976. Bogotá. Programas Editoriales, 1976. p. 205-12.
6. GANDRA, Y.R. O pré-escolar de dois a seis anos de idade e seu atendimento. Rev. Saúde puibl., S. Paulo, 15(supl): 3-8, 1981.

7. GOUVE:IA, A.J. Professoras de amanhã. um estudo da escolha ocupacional. São Paulo, Ed. Pioneira, 1970.

8. GUILFORD, J.P. Psychometric methods. New York, McGraw-Hill, 1954.

9. MACEDO, R.M.S. A organização grafo-perceptiva em pre-escolares de diferentes niveis socio-eccnómico-educacionais. São Paulo. 1973. [Tese de Dnittoramento - Faculdade de Filosofia, Ciências e Letras de São Bento da PUC].

10. MAXIMIZAÇA no uso e aproveitamento de recursos comunitários para a educação. In: Seminário de Alternativas de Desenvolvimento: Infra-estrutura e Serviços Urbanos, São Paulo, 1977. São Paulo, Secretaria de Economia e Plane. jamento, 1978, p. 249-61

11. SIEGEL, S. Estatistica não-paramétrica para as ciências do comportamento. São Paulo, McGraw-Hill do Brasil, 1975.

Recebido para publicação em 10/07/1981 Aprovado para publicasão em 17/11/1981 